\title{
More Harm than Good? A Pilot of a Motivational Interviewing Based Intervention for Increasing Readiness to Improve Nutrition in Young People Experiencing a First Episode of Psychosis
}

\author{
Emily Fulton*, Malcolm Peet ${ }^{\dagger}$ and Kevin Williamson ${ }^{\dagger}$
}

The relationship between nutrition and mental health, as well as physical health, is well known. Mental Health Services in the UK and worldwide are recognising the need for nutritional care, however eliciting a change in eating habits in patients/service users is a challenge. The effects of a 'Psych-Nutritional Intervention' (PNI) using Motivational Interviewing $(n=30)$ was compared with a 'Treatment as Usual' (TAU) involving standard nutritional advice $(n=21)$ and a control group (no nutritional support) $(n=22)$. The sample consisted of young people aged 18-35 years old with a diagnosis of psychosis, who were currently under the care of a UK specialist National Health Service (NHS) Mental Health team (Early Intervention for Psychosis Team). Nutritional knowledge was assessed at baseline across the groups. Readiness to Change eating habits was measured at baseline and a further two time points post intervention. Although there were no significant between group differences, the PNI group elicited both the most progression in terms of readiness to change eating habits, but also the most regression. The use of MI may be anti-therapeutic for those who are not ready to consider make changes to their eating habits, and this requires further investigation. Greater emphasis on the importance of assessing and selecting who is most likely to benefit from interventions is necessary.

Keywords: Eating habits; Healthy eating; Psychosis; Mental health; Motivational Interviewing

\section{Introduction}

Individuals with 'First Episode Psychosis' regularly consume less healthy diets than the general population (Williamson, Kilner \& Clibbens, 2015; Dipasquale et al., 2013). First Episode Psychosis (FEP) refers to those experiencing psychotic symptoms for the first time, in the early stage of a psychotic illness or treatment (Breitborde, Srihari \& Woods, 2009). There is a growing body of thorough and experimental research suggesting the links between food and mental health. For example, a diet rich in minerals and nutrients such as omega 3 fatty acids and antioxidants, has been linked to both the prevention and amelioration of symptoms in psychiatric illnesses such as depression and schizophrenia (Firth et al., 2017; Pawełczyk et al., 2016; Peet \& Stokes, 2005).

As a result of poor diets, patients with psychosis also have higher levels of obesity (Henderson et al., 2006), which in part explains the higher rates of physical illnesses such as diabetes, coronary heart disease and hypertension that account for the reduced life expectancy in this

\footnotetext{
* Coventry University, GB

RDASH NHS Mental Health Foundation Trust, GB

Corresponding author: Emily Fulton

(emmie.fulton@coventry.ac.uk)
}

clinical group (Mann, 2002). Equally, despite their efficacy in terms of reducing symptoms, new generation anti-psychotic drugs can result in weight gain (Russell \& Macke, 2001). Alongside a greater prevalence of smoking and a lack of exercise (Strassnig, Brar \& Ganguli, 2003), risks to physical health are high and often neglected, with treatment focused on mental health.

\section{Nutrition interventions in practice}

Despite clear argument for nutritional therapies, putting this into practice in terms of individual behavioural change, and nutritional services to support this, has been a slow process (Amani, 2007). In the setting of this study, the mental health nutritionist's goal is to assess current dietary practice using food diaries which are entered into a computer program (NetWISP Dietary Analysis software) to provide a break-down of nutritional consumption. Dietary advice regarding improved nutrition for mental and physical health, and not just weight loss, is given in the service user's home in verbal and written format (see Williamson, Kilner \& Clibbens, 2015). However, this information assumes individuals want and feel able to make changes having read or listened to the information provided. Changing health behaviour can be a challenge, particularly in this clinical group, who are further 
impeded by cognitive deficits such as poor attention and apathy resulting from the effects of medication and the illness itself (Wolf et al., 2014; Warner \& Mandiberg, 2013; Lieberman et al., 2001).

There are limited studies focusing on psychosis and nutrition, especially FEP; with the majority including a range of psychotic illnesses e.g. schizophrenia. Most of the literature focuses on weight management in obese participants, rather than improved nutrition for non-obesityrelated physical and mental health, excluding individuals of normal weight who would benefit from intervention. Although often coupled with increased physical activity, these interventions have demonstrated a change in eating habits (see Bonfioli et al., 2012), therefore lessons can be learned regarding potentially successful approaches to replicate in a study of healthy eating irrespective of participant weight. Effective psychological interventions for weight loss in psychosis include, amongst others, motivational interviewing (Menza et al., 2004; Baker et al., 2014); and dietetic and group practical sessions (Teasdale et al., 2016). Niv et al. (2014) conducted a 16-week psycho-social weight management program for individuals with schizophrenia including psychoeducation and behavioural techniques (not including motivational interviewing), but found no significant change in weight or Body Mass Index (BMI) in the intervention compared to the control group. Individuals with FEP exhibit lower levels of motivation (Lyne et al., 2012) which may explain, in part, the challenges to achieving desired outcomes in this clinical group.

One potential solution is the use of Motivational Interviewing (MI) (Miller \& Rollnick, 2002). MI is a form of face-to-face psychological therapy which incorporates a client-centred collaborative approach in eliciting behaviour change, actively resolving ambivalence without an authoritarian approach. MI encourages reflection and the generation of problem-solving strategies, emphasising personal choice and control. Interventions may consist of one brief meeting (5-10 minutes) or a formal session (1-2 hours), with the number of sessions offered depending on the goal and individual needs (Martins \& McNeil, 2009). In practice it includes using open-ended questions, building rapport, working as a collaborator, recognising the normality of mixed feelings, and providing feedback.

Meta-analyses suggest that even a brief 15 minute session of MI results in positive outcomes for a broad range of behavioural problems, in $64 \%$ of studies analysed (Rubak et al., 2005). In a systematic review and meta-analysis (Olker et al., 2016), 12 of the 14 interventions included $\mathrm{MI}$; and these were statistically more effective than a control at achieving weight loss, however no studies reached clinically significant levels (5\% weight reduction). Using MI with individuals with FEP may prove more challenging. Adherence and engagement may be compromised owing to the nature of psychotic illness and symptoms (Barkhof et al., 2006). Therefore adaptations may be necessary for this clinical group.

The aim of this pilot study was to assess the potential efficacy of a brief face-to-face motivational interviewing based intervention for enhancing 'readiness to change eating behaviours'. The intervention is based on Miller \& Rollnick (2002) and intervention manuals from previous studies (e.g. Alvarez-Jiménez et al., 2006). It was also adapted with the inclusion of behaviour change techniques presented to meet the cognitive needs of young people with first episode psychosis using visual aids and a novel workbook (see Replication Package). 'Readiness to change' refers to an individual's willingness to undertake a given behaviour in a given time period (Ceccarini et al., 2015).

\section{Methods \\ Design}

A longitudinal, part randomised controlled trial design was used for this pilot study, comprising three groups: i) Control group (who received no nutritional advice or input); ii) Usual Care (standard nutritional therapy only which included a nutritional assessment and advice on dietary change from the team nutritionist; and iii) the Motivational Interviewing (MI) intervention. Three arms were compared to establish whether usual care offered similar benefits to the intervention group. Differences in pre- and post 'Readiness to Change' healthy eating habits were compared between groups, over three time points: Time 1 (Baseline), Time 2 (one month post intervention) and Time 3 (three months post intervention). A fully randomised controlled trial was not possible due to insufficient participants in the Early Intervention for Psychosis teams (EIPT) where standard nutritional therapy is offered. One EIPT did not offer nutritional therapy at the time, therefore acted as a control group. In the other EIPT, service users were randomised to either the usual care or Intervention group. The two teams had similar models of service provision as they are managed by the same National Health Service (NHS) mental health trust, however are situated in different geographical locations in the north of England.

EIPT's are multi-disciplinary, UK-wide community based NHS mental health teams providing medical, practical and psychological care, usually within the service user's home over a three year period; to young people (usually up to 35 years of age), experiencing symptoms of psychosis (e.g. paranoid delusions, hallucinations). As part of this particular service, a nutritionist was employed to offer advice and support regarding nutrition.

\section{Sampling}

Participants were community dwelling service users of 3 EIPT's in the north of England, UK. All participants had been diagnosed with a FEP and were currently receiving psycho-social support and anti-psychotic medication from the team. They had all been seen at least once by the team nutritionist and offered dietary advice, but had not received any form of behavioural intervention to enhance healthy eating behaviour. Service users were excluded from the study if they were too unwell to take part (e.g. lacked mental capacity, as assessed by the team Psychiatrist) or became unwell after they had provided consent; 
their speech and comprehension of the English language was limited or they had learning disabilities.

\section{Procedure}

The Information sheet, questionnaires and intervention were discussed with the team and adapted and piloted with 6 ex-service users attending a carers group. All service users meeting the inclusion criteria in the control group $(\mathrm{n}=53)$, and Intervention and usual care groups ( $\mathrm{n}=97)$ were invited to take part in the study by their care co-ordinator, and given an invitation letter, information sheet and written consent form to sign. Those who agreed to take part were asked to complete a short 6-item questionnaire, with support from staff if needed, regarding their current eating habits (e.g. level of fruit, vegetable and fibre intake, consumption of high unsaturated fat, salt and refined sugary foods) to exclude those with existing healthy and well-balanced diets. The decision to exclude was made by the nutritionist who reviewed the questionnaires and consulted the service users and/ or staff for clarification if necessary. Seventy-six participants agreed to take part. Only three service users were deemed to have sufficiently balanced diets that did not require intervention, and these were screened out at this stage. All participants were blinded to the outcome of randomisation as all were told that they would receive the intervention at some point (wait-list control) over an 18 month period. In the EIPT that would include the intervention and control groups, those who had agreed to take part $(n=72)$, were given a number in a computer program database and randomly assigned to either the usual care or intervention group. The usual care and control groups received the intervention after the end of the study. All participants were given questionnaires by staff in their own homes to complete at Time 1 (T1), 1 month post intervention at Time 2 (T2), and 3 months later at Time 3 (T3). Demographic information was collected at baseline. Although weight and height were measured, BMI was not calculated and is no longer possible due to a lack of access to the raw data.

\section{Operationalisations}

\section{Manipulations}

The intervention was a brief, one-off individual faceto-face $\mathrm{MI}$ intervention utilising a series of 'Behaviour Change Techniques' (BCT's) (Michie et al., 2013). Although following the structure and principles of existing MI interventions, it was adapted into as a series of written and visual tasks within a workbook. Content included BCT's such as 'Goal Setting (behaviour)'; 'Action Planning'; 'Information about Health Consequences'; 'Instruction on how to perform the behaviour'; 'Self-monitoring of Behaviour'; 'Feedback on Behaviour' and 'Prompts/Cues'. The facilitator utilised the MI counselling style to guide the process. The intervention was developed with input from two health Psychologists, six service users and the nutritionist. Service users commented on content, presentation and ease of comprehension of the intervention, leading to revision. The intervention took place in the service user's home, lasted approximately 1 hour and was conducted by a psychologist trained in MI, recently employed by the team, who was not previously known to the service users. MI techniques were used to facilitate change talk and address the pros and cons of change. Visual aids were used to overcome problems relating to attention and memory, and to ensure fidelity so that each participant received the same content. Small goals were set and a feedback card given. Service users were telephoned by the psychologist approximately two weeks later to assess progress. In the interim period they were visited by the team nutritionist, who offered ongoing encouragement and answered questions about healthy dietary change.

\section{Measures}

Participant ethnicity, height, weight and the length of time they had been seen by the EIPT was collected at baseline to ascertain whether differences existed across the three groups that might influence the findings. Due to an unforeseen lack of access to medical information, it was not possible to collect data relating to ethnicity and the time seen by the EI team from the control group. Weight was not compared across time points as the goal of the intervention was improved healthy eating, rather than weight loss. As a result intention or 'Readiness to Change' behaviour was measured as follows:

(i) 'Readiness to Change Eating Habits Scale'. This 20Item measure includes questions based on the 5 'Stages of Change' a person is in at any given time, in terms of healthy eating (e.g. 'Pre-contemplation, Contemplation, Preparation, Action or Maintenance'). It is based on the 'Transtheoretical Model' (Prochaska \& DiClemente, 1982; 1986); and adapted from the 'University of Rhode Island Change Assessment (URICA) Weight Control Long Form' (McConnaughy, Prochaska, \& Velicer, 1983). Responses to items are recorded on a 5-point Likert scale ranging from 'Strongly Disagree' to 'Strongly Agree'. A 'Readiness to Change Eating Habits' score is calculated based on adding scores from items relating to 'active' stages (Contemplation, Planning, Action and Maintenance items) and subtracting the 'Inactive' stage item scores (Pre-contemplation). Scores range from a possible -1 to 19 , with greater scores representing a greater readiness to change behaviour. 'Variation in Readiness to Change Eating Habits' score over time was calculated by subtracting $\mathrm{T} 1$ scores from $\mathrm{T} 2$ (T2-T1) and T3 (T3-T1) scores.

(ii) 'Nutritional Knowledge Questionnaire'. This 27-Item measure (see supplementary file 1) was developed specifically for the study by the team nutritionist, to measure participants' nutritional knowledge. Although measures currently exist, none were deemed appropriate for this specific clinical group when reviewed by the nutritionist and team Psychiatrist; due to the time taken to complete, language used and level of difficulty. However aspects of existing measures were simplified. The new measure consisted of 3 subscales, including statements such as 'Eating an unhealthy diet can lead to which of the following?' and 'True' or 'False' statements such as 'To have a healthy diet everything you eat needs to be low fat'. The measure was 
piloted with 6 service users and amended accordingly. Overall scores were based on the sum of correct answers.

\section{Power Analyses}

Due to the difficulties of recruiting from this clinical population, a target of 90 service users was deemed realistic for a pilot, based on sample sizes obtained in previous research (Alvarez-Jimenez et al., 2008).

\section{Data collection period}

Data collection took place between November 2007 and March 2009.

\section{Statistical Analysis}

Data entered and analysed in the Statistical Package for the Social Sciences (SPSS). To establish whether the data was normally distributed, histograms were created, Skewness and Kurtosis calculated, and a Kolmogorov-Smirnov test performed, to ascertain whether non-parametric versions were required. Factorial ANOVA $(3 \times 3$ model) was used to look for differences in readiness to change scores over the time points, across groups. Significant differences in ethnicity across groups was assessed using the Mann-Whitney U test, and Time seen by the EIPT team was compared using an Independent Samples T-Test, as data for this variable was not available from the control group. A one-way ANOVA was conducted to establish whether there were any differences in nutritional knowledge at baseline between the 3 groups. As the analysis throughout included 3 groups, the effect size was calculated using Eta Squared $\left(\eta^{2}\right)$ rather than Cohen's d. Missing data was minimal and when present, was excluded from the analysis on an item rather than case basis. Ad hoc analyses of progression and regression in Readiness to Change scores was not possible at the time of writing, as the raw data was no longer available.

\section{Ethics}

Ethical approval was obtained from the National Research Ethics Service Committee for South Yorkshire.

\section{Results}

\section{Descriptive Statistics}

A total of 73 participants took part in the study. At Time 1, this included 30 in the intervention group, 21 in the usual care group, and 22 in the control group. A total of 50 took part at Time 1 and Time 2 , and 16 at all three time points. Attrition rates are illustrated in Table 1. Nine participants in the control group declined to complete the nutritional

Table 1: Attrition rates over the three time points.

\begin{tabular}{lrrr} 
& Time 1 & $\begin{array}{c}\text { Time 2 } \\
\text { (\% attrition } \\
\text { from Time 1) }\end{array}$ & $\begin{array}{c}\text { Time 3 } \\
\text { (\% attrition } \\
\text { from Time 1) }\end{array}$ \\
\hline Intervention & 30 & $25(17)$ & $6(76)$ \\
Usual care & 21 & $16(24)$ & $6(63)$ \\
Control & 22 & $9(59)$ & $4(56)$
\end{tabular}

knowledge questionnaire, therefore data was missing for this item (see Table 3). The Kolmogorov-Smirnov Test for normality within the data showed that the Nutrition Knowledge total scores and gender variables were not normally distributed $(p<.001)$, therefore a non-parametric test (Kruskall-Wallis) was used.

\section{Demographic characteristics}

There were no significant differences between age $(F(2,70)$ $\left.=.781, p=.462, \eta^{2}=.022\right)$, height $(F(2,50)=.541, p=$ $\left..585, \eta^{2}=.021\right)$ and gender $\left(\chi^{2}(2)=.735, p=.692, \eta^{2}=\right.$ $0.010)$; between the intervention, usual care and control groups (see Table 2). No differences existed between the intervention and usual care groups for time seen by the EI team $(t(48)=-1.214, p=.213)$ and ethnicity $(U=249.00$, $p=.057)$. The sample was predominantly 'White British' (82.4\%). Significant differences were found between the groups in terms of weight at baseline $(F(2,50)=3.468$, $\left.p=.039, \eta^{2}=.122\right)$. The mean scores indicated that the intervention group $(\mathrm{m}=87.4 \mathrm{~kg}, \mathrm{SD}=24.54)$ consisted of individuals with a greater weight than the usual care group $(\mathrm{m}=72.9 \mathrm{~kg}, \mathrm{SD}=16.28)$, and control group $(\mathrm{m}=$ $68.8 \mathrm{~kg}, \mathrm{SD}=22.17)$.

\section{Nutritional Knowledge}

There were no significant differences observed in nutritional knowledge total scores at baseline across the three groups (see Table 3) $\left(\chi^{2}(2)=.061, p=.970\right.$, $\left.\eta^{2}=.001\right)$. In general knowledge about nutrition was deemed by the nutritionist to be relatively good, although no normative comparisons were made. For example $61.9 \%$ of all participants recognised that sardines were a good source of omega 3 , and $80.6 \%$ knew that this fatty acid is important for mental as well as physical health. The majority knew that obesity can lead to heart disease and diabetes (93.5\% and $85.2 \%$ respectively), and that unhealthy foods can affect memory and concentration (80.6\%); and tiredness (lethargy) (85.5\%).

\section{Readiness to Change Eating Habits and variation in scores over time}

There were no significant differences in baseline (T1) Readiness to Change Eating Habits between the 3 groups (see Table 4) $\left(F(2,60)=.054 p=.947, \eta^{2}=.002\right)$. A $3 \times 3$ factorial ANOVA was conducted to compare the main effects of 'Time point' and 'Treatment Group' and the interaction between these factors on Readiness to Change scores. There was no significant main effect for Time $(F(2,15)=$ $.722, p=.637)$ or $\operatorname{Group}(F(2,15)=.042, p=.959)$; and no significant interaction between these two factors $(F(2,15)=$ $.292, p=.934)$. It was not possible to calculate Eta Squared scores for the factorial ANOVA as the raw and output data no longer available at the time of writing.

There were no significant differences in Variance in Readiness to Change (T3-T1) scores $(F(2,12)=.558, p=.586$, $\left.\eta^{2}=.085\right)$; and Variance in Readiness to Change scores (T2-T1) $\left(F(2,39)=.730, p=.488, \eta^{2}=.036\right)$, although the means revealed a greater increase in the Usual Care group from $\mathrm{T} 1$ to $\mathrm{T} 2$ (see Tables 5 \& $\mathbf{6}$ ). The variance in mean 
Table 2: Demographic characteristics of the three groups at T1.

\begin{tabular}{llll} 
& Intervention & Usual care & Control \\
\hline Gender (males) & 20 & 15 & 13 \\
Age (years) & $18-33$ & $18-32$ & $18-29$ \\
& $($ Mean $=23.90)$ & $($ Mean $=23.90)$ & $($ Mean $=22.50)$ \\
Ethnicity & & & \\
$\quad$ White British & 27 & 15 & $\mathrm{n} / \mathrm{a}$ \\
$\quad 3$ & 1 & $\mathrm{n} / \mathrm{a}$ \\
$\quad$ Asian British & 2 & 2 & $\mathrm{n} / \mathrm{a}$ \\
$\quad$ Asian & 1 & 1 & $\mathrm{n} / \mathrm{a}$ \\
$\quad$ Chinese & 2 & 2 & $\mathrm{n} / \mathrm{a}$ \\
$\quad$ White European & $51-126$ & $48-108$ & $42.70-108$ \\
Weight at baseline & $($ Mean $=87.36)$ & $($ Mean $=72.944)$ & $($ Mean $=68.81)$ \\
range $(\mathrm{kg})$ & $152-203$ & $155-188$ & $157-183$ \\
Height range $(\mathrm{cm})$ & $($ Mean $=173.90)$ & $($ Mean $=171.158)$ & $($ Mean $=169.20)$ \\
& $6-39$ & $5-69$ & $\mathrm{n} / \mathrm{a}$
\end{tabular}

Table 3: Comparison of mean and standard deviation (SD) Nutritional Knowledge total scores between groups at baseline (T1) and non-parametric Kruskall-Wallis Test.

\begin{tabular}{lccc} 
& N & Mean & SD \\
\hline Intervention & 30 & 19.00 & 5.942 \\
Usual care & 21 & 19.62 & 4.031 \\
Control & $13^{*}$ & 20.08 & 3.570
\end{tabular}

* Data for this outcome was missing for 9 participants in the control group who declined completion.

Table 4: Comparison of mean and standard deviation (SD) Readiness to Change Eating Habits scores between groups and across time points.

\begin{tabular}{lcccc} 
& & Time 1 & Time 2 & Time 3 \\
\hline & N & Mean (SD) & Mean (SD) & Mean (SD) \\
\hline Intervention & 30 & $9.733(2.67)$ & $11.167(1.401)$ & $11.167(5.492)$ \\
Usual care & 21 & $9.548(2.47)$ & $11.833(4.622)$ & $11.167(5.192)$ \\
Control & 22 & $9.833(2.63)$ & $11.067(4.044)$ & $11.533(4.998)$
\end{tabular}

Table 5: Comparison of the mean and standard deviation (SD) Variation in Readiness to Change (T2-T1) scores between groups.

\begin{tabular}{lrrr} 
& N & Mean & SD \\
\hline Intervention & 25 & 1.750 & 3.993 \\
Usual care & 16 & 2.539 & 4.378 \\
Control & 9 & .1667 & 2.691
\end{tabular}

Table 6: Comparison of the mean and standard deviation (SD) Variation in Readiness to Change (T3-T1) scores between groups.

\begin{tabular}{lccc} 
& N & Mean & SD \\
\hline Intervention & 6 & 2.208 & 4.010 \\
Usual care & 6 & 2.041 & 2.624 \\
Control & 4 & 4.500 & 3.969
\end{tabular}

Readiness to Change scores between T1 and T2 (T2-T1), suggests the intervention group had the greatest range of mean scores, with both the most progression in terms of readiness, and the most regression for at least one or more participants (see Figure 1).

\section{Discussion}

This study sought to pilot a brief face-to-face motivational interviewing based intervention for improving readiness to change eating habits in young people with FEP; compared to a usual (nutritional) care and control group. Thirty service users received the Intervention group, although attrition rates were high over time, and missing data present in the baseline nutritional knowledge data. Almost all the service users had a good basic nutritional knowledge at baseline, therefore it is plausible that poor eating behaviours were not principally due to a lack of knowledge, supporting the need for an intervention that offers more than nutritional information.

The intervention did not significantly increase readiness to change eating habits over and above the usual care and control groups. The brief nature of the intervention may have been insufficient to support change, and responses may be biased, illustrating what individuals hope, rather than can actually achieve. Nonetheless, an 


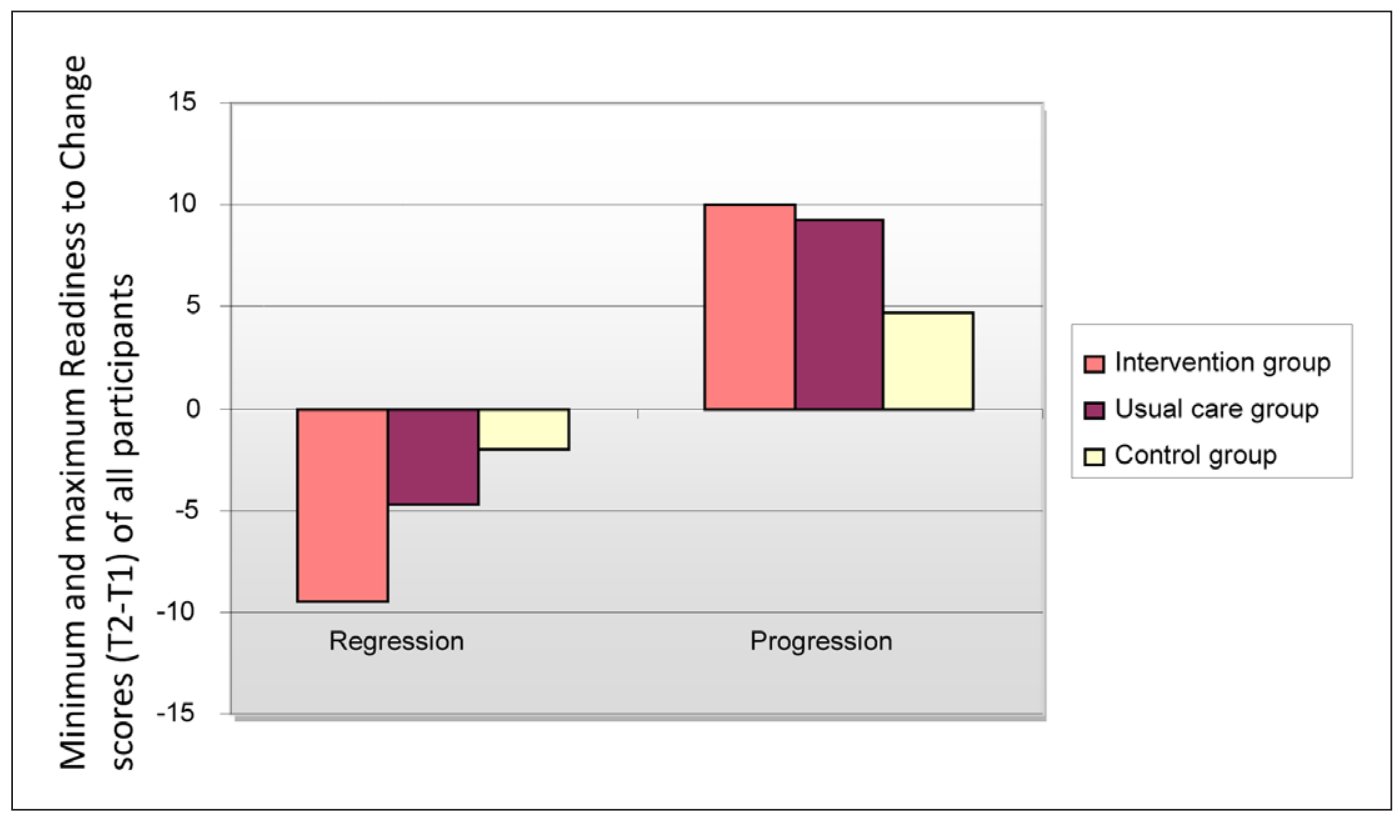

Figure 1: Graph indicating the range in degree of positive change (progression) and negative change (regression) in Readiness to Change scores between $\mathrm{T} 1$ and $\mathrm{T} 2$ by group across all participants.

interesting finding is that the intervention group had the greatest progression (or increase) in Readiness to Change between $\mathrm{T} 1$ and $\mathrm{T} 2$ for some participants (although this was only slightly greater than the usual care group); and also the most regression (reduction) for others. Therefore the intervention had the most positive effect for at least one individual, but the exact opposite for at least one other, reducing their readiness to change. However, extreme caution should be given as this represents only the range rather than frequency of scores, therefore it is not clear how many participants experienced this 'regression'. Ad hoc analyses could not be performed to explore this further. Nonetheless it does bring into question why a regression might occur for even only one participant. One potential hypothesis is that in removing the psychological and practical barriers to change, individuals lose the justification for not changing their behaviour, and if they still feel unable, or try and 'fail'; this could increase the notion that they definitely cannot achieve a change, as a self-protective mechanism. As the usual care group exhibited a similar (albeit slightly smaller) overall progression in readiness scores compared to the intervention group, but less regression, it is possible that usual care is more effective overall, as it poses less risk of discouraging some individuals, making them even less ready to change their eating habits. A more robust evaluation is required to explore this further. Clearly conclusions from this data must be cautious given the small sample size and limitations of the methodology. Caution should also be taken in generalising to all psychotic disorders (e.g. schizophrenia), as the length of illness, symptomology and medication may influence the findings.

The possibility that an intervention could lead to a negative effect on readiness to change behaviour has not been explored in the literature to date, to the authors knowledge. Nonetheless, there is evidence in relation to serious mental illness regarding factors that 'de-motivate' individuals when engaging with an intervention; which might go some way to explaining a negative effect. For example qualitative self-report studies reveal a lack of information about intervention benefits, what would happen and what was expected of participants, and perceived negative views of health professionals (Roberts \& Bailey, 2013) led to demotivation in health promotion programs. Equally, concerns about stigma, the impact of the illness and treatment effects, a lack of support, personal commitments (Roberts \& Bailey, 2011); and the effect of anxiety (Johnstone et al., 2009) may also play a role. Lifestyle interventions remain underused in mental health services (Walsh, 2011). The need to consider longer session length and higher intervention dose effect; tailoring motivational interviewing specifically to patients with mental health problems is necessary (Wong-Anuchit et al., 2018). Individuals in the later stages of treatment for psychosis have less motivation in general (Luther et al, 2015), suggesting the need to also time when interventions are offered.

\section{Limitations}

There are a number of limitations with the study. The sample was predominantly white British, and although a reasonable size given the clinical group, likely to be heterogeneous in terms of confounding variables that were not accounted for in a covariate analysis. Power to establish an effect would also have been enhanced had comparisons been made between two rather than three experimental groups; and the study lacked the true randomisation of a randomised controlled trial. The addition of a pre-post nutritional assessment of eating habits would have demonstrated whether a relationship existed between readiness to change eating habits and actual eating behaviour. The control group consisted of newer patients who may have been in an earlier stage of illness, which might have contributed to the null finding but was not assessed as data about 'Time seen by EIPT' was not collected for this 
group. An increase in readiness to change in the control group as well as the other two groups may have resulted from social desirability effects or from the prompt to change eating habits made simply by study participation.

Significant differences in weight at baseline were observed between groups. This could have affected the results due to differing levels of motivation, previous attempts to change eating behaviour which may or may not have been successful, the role of anti-psychotic induced weight gain and the cognitive impact; and the psychosocial effects of being over-weight which could have affected outcomes. As mean height was greatest in the intervention group it is possible that this explains the greater mean weight. A lack of BMI measurement is another limitation of the study. Further post-hoc analyses were also not possible as access to study data was no longer possible when the first author ceased NHS employment.

Other methodological limitations include potential randomisation bias in the control group; and due to the high attrition rates, the removal of T3 data and re-analysis may have produced more robust and alternative findings. Finally the use of a 'Readiness to Change' measure is much debated, regarding whether change can be deemed linear, with arguments that many mechanisms of change are involved; and other measures, such as self-efficacy, might be more appropriate (Lenio, 2006).

There are factors specific to FEP including the impact of symptoms and medication on attention, memory and concentration (cognitive deficits) that could impede the effectiveness of an MI intervention in this clinical group. Fiszdon et al. (2016) suggest the need to incorporate simple adjustments, including more structure, repeating and summarising content and using visual aids where possible. This is supported by Rüsch \& Corrigan (2002), who suggest the MI process is simplified into smaller steps, limiting demand on memory and attention. The intervention in this study utilised similar approaches, but would have benefitted from qualitative exploration with participants also. Given more recent knowledge about the most effective behaviour change techniques (BCT's) for healthy eating (e.g. Michie et al, 2011), which BCT's are more appropriate for this group may also have improved the outcomes. Assisting individuals to change health-related behaviours, especially in this clinical group, is a difficult and complex process. Some of the limitations in this study were indicative of conducting research in a time-, staff- and financially constrained healthcare environment, where unforeseen barriers challenged the progress of an unfunded pilot. Even with the skills of motivational interviewing at play, some participants in this study presented with low mood and apathy, consistent with the symptoms of psychosis, and high attrition rates are to be expected when conducting research with participants with severe mental illness.

The current principal focus on diet solely in the context of obesity is dangerously neglecting individuals of a healthy weight, with poor diets. Future intervention development needs to redirect targets to include this group, and consider how best to adapt methods such as $\mathrm{MI}$, to better meet their specific needs.

\section{Conclusions}

The assumption that brief MI will have either a positive or neutral effect, may be erroneous. Due to the methodological limitations of this study, no conclusions can be drawn, however further exploration of this phenomena is warranted. Future research would benefit from exploring the exact nature of using MI approaches for improved nutrition with people with first episode psychosis, and whether careful assessment of appropriateness and tailoring to overcome cognitive deficits associated with the illness and treatment is necessary, ensuring interventions are offered only to those most likely to benefit.

\section{Additional File}

The additional file for this article can be found as follows:

- The 'Full Disclosure Package' for the PNI Pilot study can be accessed via this link: https://osf.io/ p6z8r/?view_only=50081ee6c422455bbbe52a8cad c18bac.

\section{Peer Review Comments}

Health Psychology Bulletin has blind peer review, which is unblinded upon article acceptance. The editorial history of this article can be downloaded here:

- PR File 1. Editorial history. DOI: https://doi. org/10.5334/hpb.10.pr1

\section{Acknowledgements}

The authors wish to thank the service users who took part in the research, and the staff at Rotherham, Doncaster and South Humber HNS Mental Health Foundation Trust for supporting the work.

\section{Competing Interests}

The authors have no competing interests to declare.

\section{References}

Alvarez-Jiménez, M., González-Blanch, C., Vázquez-Barquero, J. L., Pérez-Iglesias, R., Martínez-García, O., Pérez-Pardal, T., Ramírez-Bonilla, M. L., \& Crespo-Facorro, B. (2006). Attenuation of antipsychotic-induced weight gain with early behavioral intervention in drug-naive first-episode psychosis patients: A randomized controlled trial. Journal of Clinical Psychiatry, 67(8), 1253-60. DOI: https://doi.org/10.4088/ JCP.v67n0812

Alvarez-Jimenez, M., Hetrick, S. E., Gonzalez-Blanch, C., Gleeson, J. F., \& McGorry, P. (2008). Non-pharmacological management of anti-psychotic-induced weight gain: Systematic review and meta-analysis of randomised controlled trials. The British Journal of Psychiatry, 193, 101-107. DOI: https://doi. org/10.1192/bjp.bp.107.042853

Amani, R. (2007). Is dietary pattern of schizophrenia patients different from healthy subjects? BMC Psychiatry, 7, 15. DOI: https://doi. org/10.1186/1471-244X-7-15 
Baker, A. L., Turner, A., Kelly, P. J., Spring, B., Callister, R., Collins, C. E., et al. (2014). Better Health Choices' by telephone: A feasibility trial of improving diet and physical activity in people diagnosed with psychotic disorders. Psychiatry research, 220(1-2), 63-70. DOI: https://doi.org/10.1016/j. psychres.2014.06.035

Barkhof, E., Haan, L. d., Meijer, C. J., Fouwels, A. J., Keet, I. P. M., Hulstijn, K. P., et al. (2006). Motivational Interviewing in Psychotic Disorders. Current Psychiatry Reviews, 2, 207-213. DOI: https://doi. org/10.2174/157340006776875932

Bonfioli, E., Berti, L., Goss, C., Muraro, F., \& Burti, L. (2012). Health promotion lifestyle interventions for weight management in psychosis: A systematic review and meta-analysis of randomized controlled trials. BioMed Central Psychiatry, 12, 1-12. DOI: https://doi.org/10.1186/1471-244X-12-78

Breitborde, N. J., Srihari, V. H., \& Woods, S. W. (2009). Review of the operational definition for first-episode psychosis. Early Interv Psychiatry, 3(4), 259-65. DOI: https://doi.org/10.1111/j.1751-7893.2009.00148.x

Ceccarini, M., Borrello, M., Pietrabissa, G., Manzoni, G. M., \& Castelnuovo, G. (2015). Assessing motivation and readiness to change for weight management and control: An in-depth evaluation of three sets of instruments. Frontiers in Psychology, 6, 511. DOI: https://doi.org/10.3389/ fpsyg.2015.00511

Dipasquale, S., Pariante, C. M., Dazzan, P., Aguglia, E., Mcguire, P., \& Mondelli, V. (2013). The dietary pattern of patients with schizophrenia: A systematic review. Journal of psychiatric research, 47(2), 197-207. DOI: https://doi.org/10.1016/j. jpsychires.2012.10.005

Firth, J., Stubbs, B., Sarris, J., Rosenbaum, S., Teasdale, S., Berk, M., et al. (2017). The effects of vitamin and mineral supplementation on symptoms of schizophrenia: A systematic review and meta-analysis. Psychological medicine, 47(9), 1515-1527. DOI: https://doi.org/10.1017/S0033291717000022

Fiszdon, J. M., Kurtz, M. M., Choi, J., Bell, M. D., \& Martino, S. (2016). Motivational Interviewing to Increase Cognitive Rehabilitation Adherence in Schizophrenia. Schizophrenia Bulletin, 42(2), 327-334. DOI: https://doi.org/10.1093/schbul/ sbv143

Henderson, D. C., Borba, C. P., Daley, T. B., Boxill, R., Nguyen, D. D., Culhane, M. A., et al. (2006). Dietary intake profile of patients with schizophrenia. Annals of Clinical Psychiatry, 18(2), 99-105. DOI: https://doi.org/10.1080/10401230600614538

Johnstone, R., Nicol, K., Donaghy, M., \& Lawrie, S. (2009). Barriers to uptake of physical activity in community-based patients with schizophrenia. Journal of Mental Health, 18(6), 523-532. DOI: https://doi. org/10.3109/09638230903111114
Lenio, J. (2006). Analysis of the Transtheoretical Model of behaviour change. Journal of Student Research, 5 , $73-87$.

Lieberman, J. A., Perkins, D., Belger, A., Chakos, M., Jarskog, F., Boteva, K., et al. (2001). The early stages of schizophrenia: Speculations on pathogenesis, pathophysiology, and therapeutic approaches. Biol. Psychiatry, 50, 884-897. DOI: https://doi. org/10.1016/S0006-3223(01)01303-8

Luther, L., Lysaker, P. H., Firmin, R. L., Breier, A., \& Vohs, J. L. (2015). Intrinsic motivation and amotivation in first episode and prolonged psychosis. Schizophrenia Research, 169(1-3), 418-422. DOI: https://doi.org/10.1016/j.schres.2015.08.040

Lyne, J., O'Donoghue, B., Owens, E., Renwick, L., Madigan, K., Kinsella, A., et al. (2012). Prevalence of item level negative symptoms in first episode psychosis diagnoses. Schizophr Res, 135(1-3), 128-133. DOI: https://doi.org/10.1016/j. schres.2012.01.004

Mann, J. I. (2002). Diet and risk of coronary heart disease and type 2 diabetes. The Lancet, 360, 783-789. DOI: https://doi.org/10.1016/ S0140-6736(02)09901-4

Martins, R. K., \& McNeil, D. W. (2009). Review of Motivational Interviewing in promoting health behaviors, Clinical Psychology Review, 29(4), 283-293. DOI: https://doi.org/10.1016/j.cpr.2009.02.001

McConnaughy, E. N., Prochaska, J. O., \& Velicer, W. F. (1983). Stages of change in psychotherapy: Measurement and sample profiles. Psychotherapy: Theory, Research and Practice, 20, 368-375. DOI: https:// doi.org/10.1037/h0090198

Menza, M., Vreeland, B., Minsky, S., Gara, M., Rigassio Radler, D., \& Sakowitz, M. (2004). Managing atypical anti-psychotic-associated weight gain: 12-month data on a multimodal weight control program. Journal of Clinical Psychiatry, 65, 471-477. DOI: https://doi.org/10.4088/JCP.v65n0404

Michie, S., Ashford, S., Sniehotta, F. F., Dombrowski, S. U., Bishop, A., \& French, D. P. (2011). A refined taxonomy of behaviour change techniques to help people change their physical activity and healthy eating behaviours: The CALORE taxonomy. Psychology \& Health, 26(11), 14791498. DOI: https://doi.org/10.1080/08870446.20 10.540664

Michie, S., Richardson, M., Johnston, M., et al. (2013). The behavior change technique taxonomy (v1) of 93 hierarchically clustered techniques: Building an international consensus for the reporting of behavior change interventions. Annals of Behavioural Medicine, 46(1). DOI: https://doi.org/10.1007/ s12160-013-9486-6

Miller, W. R., \& Rollnick, S. (2002). Motivational Interviewing ( $2^{\text {nd }}$ edition). The Guilford Press: London.

Niv, N., Cohen, A., Hamilton, A., Reist, C., \& Young, A. (2014). Effectiveness of a psychosocial weight management program for individuals with 
schizophrenia. Journal of Behavioral Health Services \& Research, 41(3), 370-380. DOI: https://doi. org/10.1007/s11414-012-9273-3

Olker, S. J., Parrott, J. S., Swarbrick, M. A., \& Spagnolo, A. B. (2016). Weight management interventions in adults with a serious mental illness: A metaanalytic review. American Journal of Psychiatric Rehabilitation, 19(4), 370-393. DOI: https://doi.org /10.1080/15487768.2016.1231643

Peet, M., \& Stokes, C. (2005). Omega-3 fatty acids in the treatment of psychiatric disorders. Drugs, 65(8), 1051-9. DOI: https://doi. org/10.2165/00003495-200565080-00002

Prochaska, J. O., \& DiClemente, C. C. (1982). Transtheoretical therapy: Toward a more integrative model of change. Psychotherapy: Theory, Research and Practice, 19(3), 276-288. DOI: https://doi.org/10.1037/ h0088437

Prochaska, J. O., \& DiClemente, C. C. (1986). Towards a comprehensive model of change, In: Miller, W. R., \& Heather, N. (eds.), Treating addictive behaviours: Processes of change, 3-27. New York: Plenum. DOI: https://doi.org/10.1007/978-1-4613-2191-0_1

Roberts, S. H., \& Bailey, J. E. (2011). Incentives and barriers to lifestyle interventions for people with severe mental illness: A narrative synthesis of quantitative, qualitative and mixed methods studies. Journal of Advanced Nursing, 67, 690-708. DOI: https://doi. org/10.1111/j.1365-2648.2010.05546.x

Roberts S. H., \& Bailey J. E. (2013). An ethnographic study of the incentives and barriers to lifestyle interventions for people with severe mental illness. Journal of Advanced Nursing, 69(11), 2514-2524. DOI: https://doi.org/10.1111/jan.12136

Rubak, S., Sandbæk, A., Lauritzen, T., \& Christensen, B. (2005). Motivational interviewing: A systematic review and meta-analysis. The British Journal of General Practice, 55(513), 305-312.

Rüsch, N., \& Corrigan, P. W. (2002). Motivational interviewing to improve insight and treatment adherence in schizophrenia. Psychiatric Rehabilitation Journal, 26(1), 23-32. DOI: https://doi. org/10.2975/26.2002.23.32
Russell, J. M., \& Mackell, J. A. (2001). Bodyweight gain associated with atypical antipsychotics: Epidemiology and therapeutic implications. CNSDrugs, 15(7), 537-51.DOI: https:// doi.org/10.2165/00023210-200115070-00004

Strassnig, M., Brar, J. S., \& Ganguli, R. (2003). Nutritional assessment of patients with schizophrenia: A preliminary study. Schizophrenia Bulletin, 29(2), 393-7. DOI: https://doi.org/10.1093/oxfordjournals.schbul.a007013

Teasdale, S. B., Ward, P. B., Rosenbaum, S., Watkins, A., Curtis, J., Kalucy, M., \& Samaras, K. (2016). A nutrition intervention is effective in improving dietary components linked to cardiometabolic risk in youth with first-episode psychosis. The British journal of nutrition, 115(11), 1987-1993. DOI: https://doi.org/10.1017/S0007114516001033

Walsh R. (2011). Lifestyle and mental health. American Psychologist, 66(7), 579-592. DOI: https://doi. org/10.1037/a0021769

Warner, R., \& Mandiberg, J. M. (2013). Social networks, support and early psychosis: Mutual support within service-user communities. Epidemiology and Psychiatric Sciences, 22(2), 151-4. DOI: https://doi. org/10.1017/S2045796012000686

Williamson, K., Kilner, K., \& Clibbens, N. (2015). A comparison of the nutrient intake of a communitydwelling first-episode psychosis cohort, aged 19-64 years, with data from the UK population. Journal of Nutritional Science, 4(e28). DOI: https://doi. org/10.1017/jns.2015.18

Wolf, D. H., Satterthwaite, T. D., Kantrowitz, J. J., Katchmar, N., Vandekar, L., Elliott, M. A., \& Ruparel, K. (2014). Amotivation in schizophrenia: Integrated assessment with behavioral, clinical, and imaging measures. Schizophr Bull, 40(6), 1328-37. DOI: https://doi.org/10.1093/schbul/sbu026

Wong-Anuchit, C., Chantamit-o-pas, C., Schneider, J. K., \& Mills, A. C. (2018). Motivational InterviewingBased Compliance/Adherence Therapy Interventions to Improve Psychiatric Symptoms of People with Severe Mental Illness: Meta-Analysis. Journal of the American Psychiatric Nurses Association. DOI https://doi.org/10.1177/1078390318761790

\footnotetext{
How to cite this article: Fulton, E., Peet, M., and Williamson, K. (2019). More Harm than Good? A Pilot of a Motivational Interviewing Based Intervention for Increasing Readiness to Improve Nutrition in Young People Experiencing a First Episode of Psychosis. Health Psychology Bulletin, 3(1), 1-9. DOl: https://doi.org/10.5334/hpb.10
}

Submitted: 12 October 2017 Accepted: 11 July 2018 Published: 02 January 2019

Copyright: (c) 2019 The Author(s). This is an open-access article distributed under the terms of the Creative Commons Attribution 4.0 International License (CC-BY 4.0), which permits unrestricted use, distribution, and reproduction in any medium, provided the original author and source are credited. See http://creativecommons.org/licenses/by/4.0/.

] $\mathrm{u}[\quad$ Health Psychology Bulletin is a peer-reviewed open access journal published by Ubiquity Press. 